Doug Geisler, Eva K. Grebel, and Dante Minniti, eds.

\title{
Dynamics of Globular Cluster Systems in Elliptical Galaxies
}

\author{
Aaron J. Romanowsky \\ Kaptyen Astronomical Institute, Postbus 800, 9700 AV Groningen, The \\ Netherlands
}

\begin{abstract}
One of the most promising avenues for exploring the dynamics of the outer parts of elliptical galaxies involves using bright discrete objects as kinematical tracers: globular clusters and planetary nebulae. As large data sets are becoming available, rigorous dynamical analyses are needed to interpret them. To this end, we have developed an extension of orbit modeling methods, which we apply to the dynamics of the giant ellipticals M87 and M49. We find that a sample of $\sim 200$ globular clusters velocities is effective at demonstrating the presence of a dark halo and providing hints about the detailed distribution of the dark matter.
\end{abstract}

\section{Introduction}

Understanding the dynamics in the outer parts of galaxies requires appropriate observational probes. For spiral galaxies, the prevalence of cold gas disks at large radii has facilitated the finding that these galaxies are generally embedded in massive dark halos. Elliptical galaxies are more difficult to probe. The standard approach is to measure the kinematics of their stars using integrated light spectroscopy - measuring rotation, velocity dispersion, and higher-order moments of the line-of-sight velocity distribution (LOSVD) such as the Gauss-Hermite moments $h_{4}$. But an elliptical's surface brightness at the large radii of interest ( $\gg$ an effective radius $R_{\text {eff }}$ ) is very low, making such observations prohibitive.

One approach especially suitable for elliptical galaxies is to measure the kinematics of bright objects within their outer parts: globular clusters (GCs) and planetary nebulae $(\mathrm{PNe})$. With a large enough data set of discrete velocities, a galaxy's mass distribution $M(r)$ can be effectively constrained. If possible, both GC and PN measurements should be used together with integrated stellar kinematics and X-ray emission analyses to put stronger combined (and somewhat disjoint) constraints on $M(r)$. Furthermore, such overlap allows one to determine $M(r)$ with one set of tracers (e.g., the stars + PNe), and then to solve for the dynamical characteristics of another set (e.g., the GCs).

This field is now coming into its own, as suitable data sets are becoming available, but careful analysis of the data is required to make robust dynamical conclusions. Firstly, the models should not assume any particular orbital structure for the system (such as isotropy). Also, they should fully exploit the information contained in the discrete velocity data: e.g., about the LOSVD shape and the escape velocity at a given radius. Many common modeling procedures, 
such as binning data in radius and velocity to estimate a projected dispersion profile $\sigma_{\mathrm{p}}(R)$, involve discarding and degrading of the available constraints.

We have developed orbit modeling methods suitable for rigorously modeling galaxies with discrete velocity data (Romanowsky \& Kochanek 2001, hereafter Paper I). We present results for two giant Virgo ellipticals, M87 and M49, using large GC data sets for both, as well as a new smaller PN data set for the latter.

\section{Observations and Modeling Methods}

For both galaxies, the distance is $\simeq 15 \mathrm{Mpc}$ and $R_{\mathrm{eff}} \simeq 100^{\prime \prime} \simeq 7 \mathrm{kpc}$.

The observational constraints on M87 and their sources are detailed in Paper I. They include the stellar surface brightness $\mu(R)$ from $R=0$. .01-745"; the integrated stellar kinematics: $\hat{\sigma}_{\mathrm{p}}(R)$ to $168^{\prime \prime}$ and $h_{4}(R)$ and $h_{6}(R)$ to $29^{\prime \prime}$; the surface density $N(R)$ of the globular cluster system (GCS) to $1351^{\prime \prime}$; and the velocities of $234 \mathrm{GCs}$ to $526^{\prime \prime}$.

The observational constraints on M49 include the stellar $\mu(R)$ to $530^{\prime \prime}$ (Kim et al. 2000); the stellar $\hat{\sigma}_{\mathrm{p}}(R)$ to $140^{\prime \prime}$ and $h_{4}(R)$ to $70^{\prime \prime}$ (Bender et al. 1994; Fisher et al. 1995); the GCS $N(R)$ to $1350^{\prime \prime}$ (Rhode \& Zepf 2001); and 144 GC velocities to $566^{\prime \prime}$ (Zepf et al. 2000). We have also obtained 24 new PN velocities to 500" using AUTOFIB2/WYFFOS at the WHT (Romanowsky et al. 2001).

The orbit modeling method pioneered by Schwarzschild (1979) is one of the most robust ways to treat elliptical galaxies. We have extended this method to incorporate data sets of discrete velocities, assuming spherical symmetry. As outlined in Paper I, this works by non-parametrically fitting a population of orbits to the data, assuming a form for the gravitational potential. We use three forms for the potential: the first corresponds to a constant mass-to-light ratio $(M / L)$ galaxy; the second is a simple dark halo model, the singular isothermal sphere; the third is a constant- $M / L$ galaxy plus a dark halo based on the cosmological simulations of Navarro, Frenk, \& White (1996, hereafter NFW).

\section{Results and Conclusions}

With the combined stellar and GCS constraints on M87, a constant- $M / L$ model is ruled out at $>5 \sigma$ significance, demonstrating conclusively the presence of a massive dark halo around this galaxy. The best mass models are NFW-based models with a mass density profile $\rho(r) \sim r^{-1.5}$ at $r \sim 200^{\prime \prime} \sim 15 \mathrm{kpc}$, and a circular velocity profile $v_{\mathrm{c}}(r)$ continuing to rise at large radii (Figure 1$)$ - nicely consistent with the mass profile derived from the galaxy's X-ray halo (Nulsen \& Böhringer 1995). At these radii, most elliptical galaxies seem to have a constant or declining $v_{\mathrm{c}}(r)$ (Gerhard et al. 2001), implying that M87 is surrounded by an extra mass component - naturally interpreted as the core of the dark halo belonging to the Virgo Cluster itself, in whose center M87 resides. Also shown in Fig. 1 are crude models that apply the spherical isotropic Jeans equations, using the stellar and GC data separately - they turn out to approximate the more rigorous results reasonably well. The stellar orbit solutions show mild radial anisotropy for $r \sim 0.1-2 \mathrm{kpc}$. The GCS anisotropy is not strongly constrained without further constraints on $M(r)$ (such as would be provided by a large set of.PN velocity data), though our NFW models are all consistent with isotropy. 


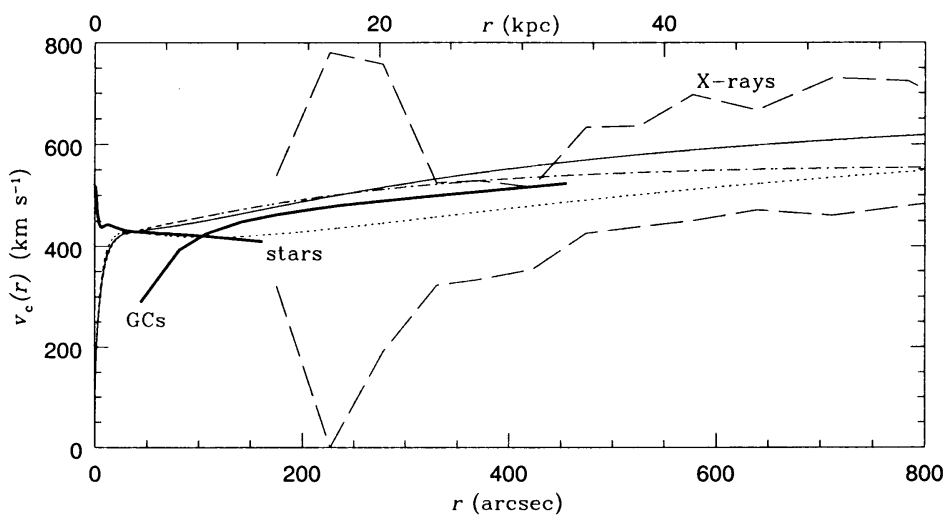

Figure 1. Circular velocity profile of M87 for different models. The heavy solid lines show estimates using the Jeans equations. The longdashed lines show X-ray confidence limits. The other lines show the best NFW-based orbit models. Note that $R_{\mathrm{eff}} \simeq 100^{\prime \prime} \simeq 7 \mathrm{kpc}$.

With preliminary results for M49, the GCs by themselves rule out a constant$M / L$ model at $2 \sigma$, and the stellar data also indicate that dark matter is required.

We have developed dynamical methods for analyzing large data sets of discrete velocities (e.g., of GCs) around elliptical galaxies, and have applied them to the giant ellipticals M87 and M49. Such data are effective at roughly determining the galaxy's mass distribution $M(r)$. The complementary analysis of determining the orbital structure of the GCS requires further constraints e.g., an independent determination of $M(r)$ using PNe. Joint models using both GCs and PNe will provide more detailed information on $M(r)$, and will furnish clues about the formational histories of the red and blue GC subsystems.

\section{References}

Bender, R., Saglia, R. P., \& Gerhard, O. E. 1994, MNRAS, 269, 785

Fisher, D., Illingworth, G., \& Franx, M. 1995, ApJ, 438, 539

Gerhard, O., Kronawitter, A., Saglia, R. P., \& Bender, R. 2001, AJ, 121, 1936

Kim, E., Lee, M. G., \& Geisler, D. 2000, MNRAS, 314, 307

Navarro, J. F., Frenk, C. S., \& White, S. D. M. 196, ApJ, 462, 563

Nulsen, P. E. J., \& Böhringer, H. 1995, MNRAS, 274, 1093

Rhode, K. L., \& Zepf, S. E. 2001, AJ, 121, 210

Romanowsky, A. J., Douglas, N. G., Arnaboldi, M., \& Kuijken, K. 2001, The ING Newsletter, 4, 23

Romanowsky, A. J., \& Kochanek, C. S. 2001, ApJ, 553, 722

Schindler, S., Binggeli, B., \& Böhringer, H. 1999, A\&A, 343, 420

Schwarzschild, M. 1979, ApJ, 232, 236

Zepf, S. E., Beasley, M. A., Bridges, T. J., Hanes, D. A., Sharples, R. M., Ashman, K. M., \& Geisler, D. 2000, AJ, 120, 2928 